\title{
Multiplication of Polynomials using Discrete Fourier Transformation
}

\author{
Krzysztof Treyderowski \\ Department of Computer Science \\ University of Gdańsk \\ Wita Stwosza 57, 80-952 Gdańsk, Poland \\ Christoph Schwarzweller \\ Department of Computer Science \\ University of Gdańsk \\ Wita Stwosza 57, 80-952 Gdańsk, Poland
}

Summary. In this article we define the Discrete Fourier Transformation for univariate polynomials and show that multiplication of polynomials can be carried out by two Fourier Transformations with a vector multiplication inbetween. Our proof follows the standard one found in the literature and uses Vandermonde matrices, see e.g. [27].

MML identifier: POLYNOM8, version: 7.8.03 4.75.958

The articles [20], [26], [28], [5], [6], [19], [12], [3], [18], [13], [25], [2], [4], [23], [8], [24], [14], [10], [11], [16], [7], [29], [22], [1], [15], [9], [21], and [17] provide the notation and terminology for this paper.

\section{Preliminaries}

The following proposition is true

(1) Let $n$ be an element of $\mathbb{N}, L$ be a unital integral domain-like non degenerated non empty double loop structure, and $x$ be an element of $L$. If $x \neq 0_{L}$, then $x^{n} \neq 0_{L}$. 
One can verify that every associative right unital add-associative right zeroed right complementable left distributive non empty double loop structure which is field-like is also integral domain-like.

The following four propositions are true:

(2) Let $L$ be an add-associative right zeroed right complementable associative commutative left unital field-like distributive non empty double loop structure and $x, y$ be elements of $L$. If $x \neq 0_{L}$ and $y \neq 0_{L}$, then $(x \cdot y)^{-1}=x^{-1} \cdot y^{-1}$.

(3) Let $L$ be an associative commutative left unital distributive field-like non empty double loop structure and $z, z_{1}$ be elements of $L$. If $z \neq 0_{L}$, then $z_{1}=\frac{z_{1} \cdot z}{z}$.

(4) Let $L$ be a left zeroed right zeroed add-associative right complementable non empty double loop structure, $m$ be an element of $\mathbb{N}$, and $s$ be a finite sequence of elements of $L$. Suppose len $s=m$ and for every element $k$ of $\mathbb{N}$ such that $1 \leq k$ and $k \leq m$ holds $s_{k}=1_{L}$. Then $\sum s=m \cdot 1_{L}$.

(5) Let $L$ be an add-associative right zeroed right complementable associative commutative left unital distributive field-like non empty double loop structure, $s$ be a finite sequence of elements of $L$, and $q$ be an element of $L$. Suppose $q \neq 1_{L}$ and for every natural number $i$ such that $1 \leq i$ and $i \leq$ len $s$ holds $s(i)=q^{i-{ }^{\prime} 1}$. Then $\sum s=\frac{1_{L}-q^{\text {len }} s}{1_{L}-q}$.

Let $L$ be a unital non empty double loop structure and let $m$ be an element of $\mathbb{N}$. The functor $m_{L}$ yielding an element of $L$ is defined as follows:

(Def. 1) $m_{L}=m \cdot 1_{L}$.

Next we state several propositions:

(6) Let $L$ be a field and $m, n, k$ be elements of $\mathbb{N}$. Suppose $m>0$ and $n>0$. Let $M_{1}$ be a matrix over $L$ of dimension $m \times n$ and $M_{2}$ be a matrix over $L$ of dimension $n \times k$. Then $\left(m_{L} \cdot M_{1}\right) \cdot M_{2}=m_{L} \cdot\left(M_{1} \cdot M_{2}\right)$.

(7) Let $L$ be a non empty zero structure, $p$ be an algebraic sequence of $L$, and $i$ be an element of $\mathbb{N}$. If $p(i) \neq 0_{L}$, then len $p \geq i+1$.

(8) For every non empty zero structure $L$ and for every algebraic sequence $s$ of $L$ such that len $s>0$ holds $s($ len $s-1) \neq 0_{L}$.

(9) Let $L$ be an add-associative right zeroed right complementable distributive commutative associative left unital integral domain-like non empty double loop structure and $p, q$ be polynomials of $L$. If len $p>0$ and len $q>0$, then len $(p * q) \leq$ len $p+$ len $q$.

(10) Let $L$ be an associative non empty double loop structure, $k, l$ be elements of $L$, and $s_{1}$ be a sequence of $L$. Then $k \cdot\left(l \cdot s_{1}\right)=(k \cdot l) \cdot s_{1}$. 


\section{Multiplication of Algebraic Sequences}

Let $L$ be a non empty double loop structure and let $m_{1}, m_{2}$ be sequences of $L$. The functor $m_{1} \cdot m_{2}$ yields a sequence of $L$ and is defined as follows:

(Def. 2) For every element $i$ of $\mathbb{N}$ holds $\left(m_{1} \cdot m_{2}\right)(i)=m_{1}(i) \cdot m_{2}(i)$.

Let $L$ be an add-associative right zeroed right complementable left distributive non empty double loop structure and let $m_{1}, m_{2}$ be algebraic sequences of $L$. Observe that $m_{1} \cdot m_{2}$ is finite-Support.

We now state two propositions:

(11) Let $L$ be an add-associative right zeroed right complementable distributive non empty double loop structure and $m_{1}, m_{2}$ be algebraic sequences of $L$. Then $\operatorname{len}\left(m_{1} \cdot m_{2}\right) \leq \min \left(\operatorname{len} m_{1}\right.$, len $\left.m_{2}\right)$.

(12) Let $L$ be an add-associative right zeroed right complementable distributive integral domain-like non empty double loop structure and $m_{1}, m_{2}$ be algebraic sequences of $L$. If len $m_{1}=\operatorname{len} m_{2}$, then len $\left(m_{1} \cdot m_{2}\right)=\operatorname{len} m_{1}$.

\section{Powers in Double Loop Structures}

Let $L$ be an associative commutative left unital distributive field-like non empty double loop structure, let $a$ be an element of $L$, and let $i$ be an integer. The functor $a^{i}$ yielding an element of $L$ is defined as follows:

(Def. 3) $\quad a^{i}=\left\{\begin{array}{l}\operatorname{power}_{L}(a, i), \text { if } 0 \leq i, \\ \operatorname{power}_{L}(a,|i|)^{-1}, \text { otherwise. }\end{array}\right.$

Next we state a number of propositions:

(13) Let $L$ be an associative commutative left unital distributive field-like non empty double loop structure and $x$ be an element of $L$. Then $x^{0}=1_{L}$.

(14) Let $L$ be an associative commutative left unital distributive field-like non empty double loop structure and $x$ be an element of $L$. Then $x^{1}=x$.

(15) Let $L$ be an associative commutative left unital distributive field-like non empty double loop structure and $x$ be an element of $L$. Then $x^{-1}=x^{-1}$.

(16) Let $L$ be an associative commutative left unital distributive field-like non degenerated non empty double loop structure and $i$ be an integer. Then $\left(1_{L}\right)^{i}=1_{L}$

(17) Let $L$ be an associative commutative left unital distributive field-like non empty double loop structure, $x$ be an element of $L$, and $n$ be an element of $\mathbb{N}$. Then $x^{n+1}=x^{n} \cdot x$ and $x^{n+1}=x \cdot x^{n}$.

(18) Let $L$ be an add-associative right zeroed right complementable associative commutative left unital distributive field-like non degenerated non empty double loop structure, $i$ be an integer, and $x$ be an element of $L$. If $x \neq 0_{L}$, then $\left(x^{i}\right)^{-1}=x^{-i}$. 
(19) For every field $L$ and for every integer $j$ and for every element $x$ of $L$ such that $x \neq 0_{L}$ holds $x^{j+1}=x^{j} \cdot x^{1}$.

(20) For every field $L$ and for every integer $j$ and for every element $x$ of $L$ such that $x \neq 0_{L}$ holds $x^{j-1}=x^{j} \cdot x^{-1}$.

(21) For every field $L$ and for all integers $i, j$ and for every element $x$ of $L$ such that $x \neq 0_{L}$ holds $x^{i} \cdot x^{j}=x^{i+j}$.

(22) Let $L$ be a field-like associative unital add-associative right zeroed right complementable left distributive commutative non degenerated non empty double loop structure, $k$ be an element of $\mathbb{N}$, and $x$ be an element of $L$. If $x \neq 0_{L}$, then $\left(x^{-1}\right)^{k}=x^{-k}$.

(23) Let $L$ be a field and $x$ be an element of $L$. Suppose $x \neq 0_{L}$. Let $i, j, k$ be natural numbers. Then $x^{(i-1) \cdot(k-1)} \cdot x^{-(j-1) \cdot(k-1)}=x^{(i-j) \cdot(k-1)}$.

(24) Let $L$ be an associative commutative left unital distributive field-like non empty double loop structure, $x$ be an element of $L$, and $n, m$ be elements of $\mathbb{N}$. Then $x^{n \cdot m}=\left(x^{n}\right)^{m}$.

(25) For every field $L$ and for every element $x$ of $L$ such that $x \neq 0_{L}$ and for every integer $i$ holds $\left(x^{-1}\right)^{i}=\left(x^{i}\right)^{-1}$.

(26) For every field $L$ and for every element $x$ of $L$ such that $x \neq 0_{L}$ and for all integers $i, j$ holds $x^{i \cdot j}=\left(x^{i}\right)^{j}$.

(27) Let $L$ be an associative commutative left unital distributive field-like non empty double loop structure, $x$ be an element of $L$, and $i, k$ be elements of $\mathbb{N}$. If $1 \leq k$, then $x^{i \cdot(k-1)}=\left(x^{i}\right)^{k-1}$.

\section{Conversion between Algebraic Sequences and Matrices}

Let $m$ be a natural number, let $L$ be a non empty zero structure, and let $p$ be an algebraic sequence of $L$. The functor $\operatorname{mConv}(p, m)$ yielding a matrix over $L$ of dimension $m \times 1$ is defined as follows:

(Def. 4) For every natural number $i$ such that $1 \leq i$ and $i \leq m$ holds $(\mathrm{mConv}(p, m))_{i, 1}=p(i-1)$.

We now state two propositions:

(28) Let $m$ be a natural number. Suppose $m>0$. Let $L$ be a non empty zero structure and $p$ be an algebraic sequence of $L$. Then len $m \operatorname{Conv}(p, m)=m$ and width $m \operatorname{Conv}(p, m)=1$ and for every natural number $i$ such that $i<m$ holds $(m \operatorname{Conv}(p, m))_{i+1,1}=p(i)$.

(29) Let $m$ be a natural number. Suppose $m>0$. Let $L$ be a non empty zero structure, $a$ be an algebraic sequence of $L$, and $M$ be a matrix over $L$ of dimension $m \times 1$. Suppose that for every natural number $i$ such that $i<m$ holds $M_{i+1,1}=a(i)$. Then $\operatorname{mConv}(a, m)=M$. 
Let $L$ be a non empty zero structure and let $M$ be a matrix over $L$. The functor aConv $M$ yielding an algebraic sequence of $L$ is defined by the conditions (Def. 5).

(Def. 5)(i) For every natural number $i$ such that $i<\operatorname{len} M$ holds $(\operatorname{aConv} M)(i)=$ $M_{i+1,1}$, and

(ii) for every natural number $i$ such that $i \geq \operatorname{len} M$ holds $(\operatorname{aConv} M)(i)=$ $0_{L}$.

\section{Primitive Roots, DFt and Vandermonde Matrix}

Let $L$ be a unital non empty double loop structure, let $x$ be an element of $L$, and let $n$ be an element of $\mathbb{N}$. We say that $x$ is primitive root of degree $n$ if and only if:

(Def. 6) $\quad n \neq 0$ and $x^{n}=1_{L}$ and for every element $i$ of $\mathbb{N}$ such that $0<i$ and $i<n$ holds $x^{i} \neq 1_{L}$.

We now state three propositions:

(30) Let $L$ be a unital add-associative right zeroed right complementable right distributive non degenerated non empty double loop structure and $n$ be an element of $\mathbb{N}$. Then $0_{L}$ is !not primitive root of degree $n$.

(31) Let $L$ be an add-associative right zeroed right complementable associative commutative unital distributive field-like non degenerated non empty double loop structure, $m$ be an element of $\mathbb{N}$, and $x$ be an element of $L$. If $x$ is primitive root of degree $m$, then $x^{-1}$ is primitive root of degree $m$.

(32) Let $L$ be an add-associative right zeroed right complementable associative commutative left unital distributive field-like non degenerated non empty double loop structure, $m$ be an element of $\mathbb{N}$, and $x$ be an element of $L$. Suppose $x$ is primitive root of degree $m$. Let $i, j$ be natural numbers. If $1 \leq i$ and $i \leq m$ and $1 \leq j$ and $j \leq m$ and $i \neq j$, then $x^{i-j} \neq 1_{L}$.

Let $m$ be a natural number, let $L$ be a unital non empty double loop structure, let $p$ be a polynomial of $L$, and let $x$ be an element of $L$. The functor $\operatorname{DFT}(p, x, m)$ yielding an algebraic sequence of $L$ is defined by the conditions (Def. 7).

(Def. 7)(i) For every element $i$ of $\mathbb{N}$ such that $i<m$ holds $(\operatorname{DFT}(p, x, m))(i)=$ $\operatorname{eval}\left(p, x^{i}\right)$, and

(ii) for every element $i$ of $\mathbb{N}$ such that $i \geq m$ holds $(\operatorname{DFT}(p, x, m))(i)=0_{L}$.

The following propositions are true:

(33) Let $m$ be a natural number, $L$ be a unital non empty double loop structure, and $x$ be an element of $L$. Then $\operatorname{DFT}(\mathbf{0} . L, x, m)=\mathbf{0}$. $L$.

(34) Let $m$ be a natural number, $L$ be a field, $p, q$ be polynomials of $L$, and $x$ be an element of $L$. Then $\operatorname{DFT}(p, x, m) \cdot \operatorname{DFT}(q, x, m)=\operatorname{DFT}(p * q, x, m)$. 
Let $L$ be an associative commutative left unital distributive field-like non empty double loop structure, let $m$ be a natural number, and let $x$ be an element of $L$. The functor Vandermonde $(x, m)$ yielding a matrix over $L$ of dimension $m$ is defined as follows:

(Def. 8) For all natural numbers $i, j$ such that $1 \leq i$ and $i \leq m$ and $1 \leq j$ and $j \leq m$ holds (Vandermonde $(x, m))_{i, j}=x^{(i-1) \cdot(j-1)}$.

Let $L$ be an associative commutative left unital distributive field-like non empty double loop structure, let $m$ be a natural number, and let $x$ be an element of $L$. We introduce $\operatorname{VM}(x, m)$ as a synonym of $\operatorname{Vandermonde}(x, m)$.

One can prove the following propositions:

(35) Let $L$ be a field and $m, n$ be natural numbers. Suppose $m>0$. Let $M$ be a matrix over $L$ of dimension $m \times n$. Then $\left(\begin{array}{lll}1 & & 0 \\ & \ddots & \\ 0 & & 1\end{array}\right)_{L}^{m \times m} \cdot M=M$.

(36) Let $L$ be a field and $m$ be an element of $\mathbb{N}$. Suppose $0<m$. Let $u$, $v, u_{1}$ be matrices over $L$ of dimension $m$. Suppose that for all natural numbers $i, j$ such that $1 \leq i$ and $i \leq m$ and $1 \leq j$ and $j \leq m$ holds $(u \cdot v)_{i, j}=m_{L} \cdot\left(u_{1}\right)_{i, j}$. Then $u \cdot v=m_{L} \cdot u_{1}$.

(37) Let $L$ be a field, $x$ be an element of $L, s$ be a finite sequence of elements of $L$, and $i, j, m$ be elements of $\mathbb{N}$. Suppose that $x$ is primitive root of degree $m$ and $1 \leq i$ and $i \leq m$ and $1 \leq j$ and $j \leq m$ and len $s=m$ and for every natural number $k$ such that $1 \leq k$ and $k \leq m$ holds $s_{k}=x^{(i-j) \cdot(k-1)}$. Then $\left(\operatorname{VM}(x, m) \cdot \operatorname{VM}\left(x^{-1}, m\right)\right)_{i, j}=\sum s$.

(38) Let $L$ be a field, $m, i, j$ be elements of $\mathbb{N}$, and $x$ be an element of $L$. Suppose $i \neq j$ and $1 \leq i$ and $i \leq m$ and $1 \leq j$ and $j \leq m$ and $x$ is primitive root of degree $m$. Then $\left(\operatorname{VM}(x, m) \cdot \operatorname{VM}\left(x^{-1}, m\right)\right)_{i, j}=0_{L}$.

(39) Let $L$ be a field and $m$ be an element of $\mathbb{N}$. Suppose $m>0$. Let $x$ be an element of $L$. If $x$ is primitive root of degree $m$, then $\operatorname{VM}(x, m)$. $\operatorname{VM}\left(x^{-1}, m\right)=m_{L} \cdot\left(\begin{array}{ccc}1 & & 0 \\ & \ddots & \\ 0 & & 1\end{array}\right)_{L}^{m \times m}$.

(40) Let $L$ be a field, $m$ be an element of $\mathbb{N}$, and $x$ be an element of $L$. If $m>0$ and $x$ is primitive root of degree $m$, then $\operatorname{VM}(x, m) \cdot \operatorname{VM}\left(x^{-1}, m\right)=$ $\operatorname{VM}\left(x^{-1}, m\right) \cdot \operatorname{VM}(x, m)$. 


\section{DFt-Multiplication of Polynomials}

We now state four propositions:

(41) Let $L$ be a field, $p$ be a polynomial of $L$, and $m$ be an element of $\mathbb{N}$. Suppose $m>0$ and len $p \leq m$. Let $x$ be an element of $L$ and $i$ be an element of $\mathbb{N}$. If $i<m$, then $(\operatorname{DFT}(p, x, m))(i)=(\operatorname{VM}(x, m) \cdot \operatorname{mConv}(p, m))_{i+1,1}$.

(42) Let $L$ be a field, $p$ be a polynomial of $L$, and $m$ be a natural number. If $0<m$ and len $p \leq m$, then for every element $x$ of $L$ holds $\operatorname{DFT}(p, x, m)=$ $\operatorname{aConv}(\operatorname{VM}(x, m) \cdot \mathrm{m} \operatorname{Conv}(p, m))$.

(43) Let $L$ be a field, $p, q$ be polynomials of $L$, and $m$ be an element of $\mathbb{N}$. Suppose $m>0$ and len $p \leq m$ and len $q \leq m$. Let $x$ be an element of $L$. If $x$ is primitive root of degree $2 \cdot m$, then $\operatorname{DFT}\left(\operatorname{DFT}(p * q, x, 2 \cdot m), x^{-1}, 2 \cdot m\right)=$ $(2 \cdot m)_{L} \cdot(p * q)$.

(44) Let $L$ be a field, $p, q$ be polynomials of $L$, and $m$ be an element of $\mathbb{N}$. Suppose $m>0$ and len $p \leq m$ and len $q \leq m$. Let $x$ be an element of $L$. Suppose $x$ is primitive root of degree $2 \cdot m$. If $(2 \cdot m)_{L} \neq 0_{L}$, then $\left((2 \cdot m)_{L}\right)^{-1} \cdot \operatorname{DFT}\left(\operatorname{DFT}(p, x, 2 \cdot m) \cdot \operatorname{DFT}(q, x, 2 \cdot m), x^{-1}, 2 \cdot m\right)=p * q$.

\section{REFERENCES}

[1] Grzegorz Bancerek. The fundamental properties of natural numbers. Formalized Mathematics, 1(1):41-46, 1990.

[2] Grzegorz Bancerek and Krzysztof Hryniewiecki. Segments of natural numbers and finite sequences. Formalized Mathematics, 1(1):107-114, 1990.

[3] Czesław Byliński. Binary operations. Formalized Mathematics, 1(1):175-180, 1990.

[4] Czesław Byliński. Finite sequences and tuples of elements of a non-empty sets. Formalized Mathematics, 1(3):529-536, 1990.

[5] Czesław Byliński. Functions and their basic properties. Formalized Mathematics, 1(1):5565, 1990.

[6] Czesław Byliński. Functions from a set to a set. Formalized Mathematics, 1(1):153-164, 1990.

[7] Katarzyna Jankowska. Matrices. Abelian group of matrices. Formalized Mathematics, 2(4):475-480, 1991.

[8] Eugeniusz Kusak, Wojciech Leończuk, and Michał Muzalewski. Abelian groups, fields and vector spaces. Formalized Mathematics, 1(2):335-342, 1990.

[9] Robert Milewski. The evaluation of polynomials. Formalized Mathematics, 9(2):391-395, 2001.

[10] Robert Milewski. Fundamental theorem of algebra. Formalized Mathematics, 9(3):461470, 2001.

[11] Robert Milewski. The ring of polynomials. Formalized Mathematics, 9(2):339-346, 2001.

[12] Michał Muzalewski. Construction of rings and left-, right-, and bi-modules over a ring. Formalized Mathematics, 2(1):3-11, 1991.

[13] Michał Muzalewski and Wojciech Skaba. From loops to abelian multiplicative groups with zero. Formalized Mathematics, 1(5):833-840, 1990.

[14] Michał Muzalewski and Lesław W. Szczerba. Construction of finite sequences over ring and left-, right-, and bi-modules over a ring. Formalized Mathematics, 2(1):97-104, 1991.

[15] Takaya Nishiyama and Yasuho Mizuhara. Binary arithmetics. Formalized Mathematics, 4(1):83-86, 1993.

[16] Jan Popiołek. Real normed space. Formalized Mathematics, 2(1):111-115, 1991.

[17] Konrad Raczkowski. Integer and rational exponents. Formalized Mathematics, 2(1):125$130,1991$. 
[18] Christoph Schwarzweller. The binomial theorem for algebraic structures. Formalized Mathematics, 9(3):559-564, 2001.

[19] Andrzej Trybulec. Subsets of complex numbers. To appear in Formalized Mathematics.

[20] Andrzej Trybulec. Tarski Grothendieck set theory. Formalized Mathematics, 1(1):9-11, 1990.

[21] Andrzej Trybulec. Tuples, projections and Cartesian products. Formalized Mathematics, 1(1):97-105, 1990.

[22] Michał J. Trybulec. Integers. Formalized Mathematics, 1(3):501-505, 1990.

[23] Wojciech A. Trybulec. Groups. Formalized Mathematics, 1(5):821-827, 1990.

[24] Wojciech A. Trybulec. Pigeon hole principle. Formalized Mathematics, 1(3):575-579, 1990.

[25] Wojciech A. Trybulec. Vectors in real linear space. Formalized Mathematics, 1(2):291$296,1990$.

[26] Zinaida Trybulec. Properties of subsets. Formalized Mathematics, 1(1):67-71, 1990.

[27] J. von zur Gathen and J. Gerhard Modern Computer Algebra. Cambridge University Press, 1999.

[28] Edmund Woronowicz. Relations and their basic properties. Formalized Mathematics, 1(1):73-83, 1990.

[29] Katarzyna Zawadzka. The product and the determinant of matrices with entries in a field. Formalized Mathematics, 4(1):1-8, 1993.

Received October 12, 2006 